\section{Effect of manuka honey on 5-fluorouracil chemosensitivity in colonspheres enriched with cancer stem (-like) cells}

\author{
Danila Cianciosi, ${ }^{1}$ Lucia Regolo, ${ }^{1}$ \\ Maurizio Battino, ${ }^{1,2}$ \\ Francesca Giampieri, 1,3
}

1Department of Clinical Sciences, Polytechnic University of Marche, Ancona, Italy; ${ }^{2}$ International Research Center for Food Nutrition and Safety, Jiangsu University, Zhenjiang, China; 3Department of Biochemistry, Faculty of Sciences, King Abdulaziz University, Jeddah, Saudi Arabia

\begin{abstract}
Manuka honey $(\mathrm{MH})$ has an in vitro chemosensitizing effect against 5Fluorouracil $(5-\mathrm{Fu})$ in colonspheres enriched with cancer stem (-like) cells (CSCs-like); these interesting findings, although preliminary, suggest a potential synergistic action of $\mathrm{MH}$ with $5-\mathrm{Fu}$, which could be useful in deepening the studies on natural compounds to be used in association with conventional medical therapies.
\end{abstract}

\section{Introduction}

Colorectal Cancer (CRC) represents worldwide the $3^{\text {rd }}$ cancer for incidence and the $2^{\text {nd }}$ for mortality. ${ }^{1}$ The low efficacy of the pharmacological treatments used, among which 5-Fu is the most common, is largely attributable to the phenomenon of chemoresistance typical of a rare tumor subpopulation: the CSCs. ${ }^{2}$ In recent years, research has been focusing on the study of various compounds, including natural ones, that have a chemosensitizing effect on common chemotherapeutic drugs. In this context, honey represents a natural food product known to modulate various biological activities and prevent various diseases, including cancer. ${ }^{3}$ The main objective of the present work was to evaluate the in vitro effect of $\mathrm{MH}$ and its combination with 5-Fu on CSCs-like deriving from colon adenocarcinoma cell line, enriched through the sphere formation assay, a 3D tumor model that better mimics what really happens in vivo. 4

\section{Materials and Methods}

Two different cell lines were used: human colon adenocarcinoma cell line and human colon fibroblast cell line.

The ability of $\mathrm{MH}$ alone and in combination with 5-Fu to decrease some morphological and physical parameters of colonspheres enriched with CSCs-like.

The effect of $\mathrm{MH}$ alone and in combination with 5-Fu on ROS intracellular accumulation and apoptotic rate in colonspheres enriched with CSCs-like was evaluated with cytometry.

Western Blot and Real-Time PCR analysis were used to evaluate the effect of $\mathrm{MH}$ alone and in combination with 5-Fu on colonspheres enriched with CSCs-like regarding different peculiar characteristics and processes of CSCs.

Self-renewal and migration assay were used for evaluating the effect of $\mathrm{MH}$ alone and in combination with $5-\mathrm{Fu}$ on colonspheres enriched with CSCs-like on selfrenewal ability and migration capacity.

Statistical analysis was performed using STATISTICA software (Statsoft Inc., Tulsa, OK, USA). For the significant differences are used letters that were acquired using one-way analysis of variance (ANOVA) followed by Tukey's honest significant difference post hoc test $(p<0.05)$.

\section{Results}

$\mathrm{MH}$ alone and more in combination with 5-Fu was able to decrease some morphological and physical parameters of colonspheres without exerting any cytotoxic effect on healthy colon cells.
Correspondence:Francesca Giampieri, Department of Clinical Sciences, Polytechnic University of Marche, Ancona, Italy; Department of Biochemistry, Faculty of Sciences, King Abdulaziz University, Jeddah, Saudi Arabia.

E-mail: f.giampieri@staff.univpm.it

Key words: 5-Fluoruracil; cancer; honey; spheroids; stemness.

Disclosures: Authors have nothing to disclose.

Conference presentation: This paper was presented at the Third Centro 3R Annual Meeting - L'era delle 3R: modelli in silico, in vitro e in vivo per promuovere la ricerca traslazionale 30 September - 1 October 2021, Evento online organizzato dal Politecnico di Torino.

Received for publication: 9 July 2021.

Accepted for publication: 7 September 2021.

This work is licensed under a Creative Commons Attribution NonCommercial 4.0 License (CC BY-NC 4.0).

(c) Copyright: the Author(s), 2021

Licensee PAGEPress, Italy

Biomedical Science and Engineering 2021; 4(s1):163 doi:10.4081/bse.2021.163

$\mathrm{MH}$ and its combination with 5-Fu induced intracellular accumulation of ROS associated with increased apoptosis rate.

$\mathrm{MH}$ in combination with 5-Fu was able to downregulate some gene expression strictly associated with a higher chemoresistance.

MH was able to enhance the effect of 5$\mathrm{Fu}$ in the downregulation of one of the main targets of 5-Fu.
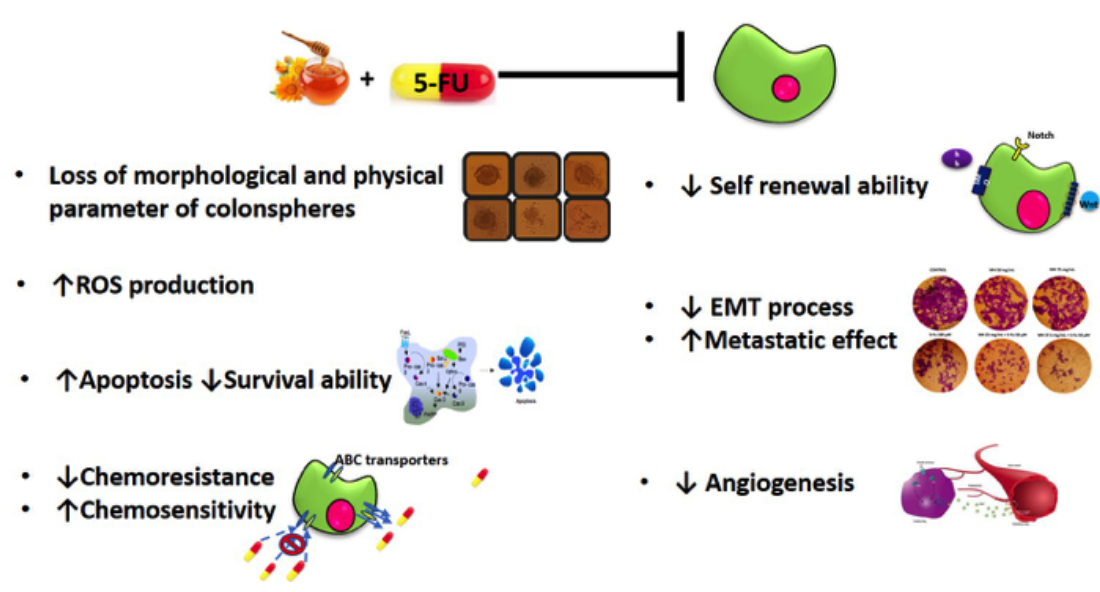

Figure 1. Summary of the effects showed by MH and 5-Fu on colon CSCs-like. 
- $\quad$ MH was able to increase the effect of 5$\mathrm{Fu}$ on the self-renewal ability.

- $\mathrm{MH}$, especially in combination with 5$\mathrm{Fu}$, was able to reduce the migration capacity of CSCs-like and some proangiogenic factors.

\section{Discussion and Conclusions}

These results indicate that $\mathrm{MH}$ has an in vitro chemosensitizing effect against $5-\mathrm{Fu}$ on colon CSCs-like (Figure 1); these interesting findings, although preliminary, suggest a potential synergistic action of $\mathrm{MH}$ with 5-Fu, which could be useful in deepening the studies on natural compounds to be used in association with conventional medical therapies which, in addition to increase the effectiveness of 5-Fu, also may decrease its side effects. The main limitation of this study is related to the bioavailability and bioaccessibility of phenolic compounds. This limitation can be a starting point for subsequent studies involving in vitro simulated digestion processes. The treatment with the digested fraction could allow an approach to a physiological condition closer to the real one.

\section{References}

1. International Agency for Research on
Cancer - World Health Organization. Global Cancer Observatory. GLOBOCAN 2020. Available from: https://gco.iarc.fr/

2. Rycaj K, Tang DG. Cancer stem cells and radioresistance. Int $\mathrm{J}$ Radiat Biol 2014;90:615-21.

3. Cianciosi D, Forbes-Hernández TY, Afrin S, et al. Phenolic Compounds in Honey and Their Associated Health Benefits: A Review. Molecules 2018;23:2322.

4. Zanoni M, Piccinini F, Arienti C, et al. 3D tumor spheroid models for in vitro therapeutic screening: a systematic approach to enhance the biological relevance of data obtained. Sci Rep 2016;6:19103. 